\title{
Role of steroid receptor and coregulator mutations in hormone-dependent cancers
}

\author{
Anna C. Groner ${ }^{1,2,3}$ and Myles Brown ${ }^{1,2}$ \\ 'Department of Medical Oncology and '2enter for Functional Cancer Epigenetics, Dana-Farber Cancer Institute, Harvard Medical School, Boston, Massachusetts, USA. \\ ${ }^{3}$ Department of Biosystems Science and Engineering, ETH Zurich, Basel, Switzerland.
}

\begin{abstract}
Steroid hormones mediate critical lineage-specific developmental and physiologic responses. They function by binding their cognate receptors, which are transcription factors that drive specific gene expression programs. The requirement of most prostate cancers for androgen and most breast cancers for estrogen has led to the development of endocrine therapies that block the action of these hormones in these tumors. While initial endocrine interventions are successful, resistance to therapy often arises. We will review how steroid receptor-dependent genomic signaling is affected by genetic alterations in endocrine therapy resistance. The detailed understanding of these interactions will not only provide improved treatment options to overcome resistance, but, in the future, will also be the basis for implementing precision cancer medicine approaches.
\end{abstract}

\section{Introduction}

The transcriptional program of a cell is shaped by its transcription factor (TF) repertoire and its genetic makeup. We summarize molecular mechanisms involved in creating steroid receptordriven gene expression programs in hormone-dependent cancers and will elucidate the interplay between steroid receptor-dependent transcription and genetic alterations that contribute to endocrine therapy resistance. We focus on processes that share molecular similarities in prostate cancer (PCa) and breast cancer (BCa). This is based on the idea that the androgen receptor (AR) and the estrogen receptor (ER) $\alpha$ are related TFs and have similar functions in driving both primary and recurrent disease. Other cancers, such as endometrial cancers, are also hormone driven but will not be covered here due to space constraints. We highlight molecular mechanisms that underlie the adaptation of the transcriptional or genomic activity of AR and ER in endocrine therapy-resistant PCa and $\mathrm{BCa}$ and discuss how genetic alterations may influence this process. Furthermore, we discuss how understanding the mode of action of specific genetic changes might provide improved and more precise treatments of endocrine therapy-resistant cancers.

\section{The clinical problem of endocrine therapy resistance}

Targeting AR in PCa. PCa remains one of the most common causes of male cancer deaths worldwide (1). In 2017, approximately 161,360 men will be diagnosed with PCa in the United States - of whom an estimated 26,730 will die from the disease (2). Nearly all diagnosed cases are localized (3) and are treated by surgery or radiotherapy. While these treatments are initially effective, many patients rapidly relapse and develop recurrent metastatic disease, which is often fatal, as evidenced by a five-year survival rate of $28 \%$ (4).

Conflict of interest: M. Brown is a consultant for GTx, Inc., and Novartis. He receives research support from Novartis.

Reference information: / Clin Invest. 2017;127(4):1126-1135.

https://doi.org/10.1172/JCI88885.
Locally advanced and metastatic PCa therapy aims to reduce serum androgen levels and inhibit AR function. Androgen deprivation therapy (ADT) has been the mainstay treatment for advanced PCa for many years (5). Current first-line ADT suppresses testicular androgen secretion (6). Additional treatments include adrenal androgen synthesis inhibitors, such as abiraterone $(7,8)$, and antagonists that prevent androgen/AR binding, such as enzalutamide (9). Unfortunately, most patients with advanced disease develop resistance to AR inhibition and progress to a lethal, endocrine therapy-resistant stage termed castration-resistant $\mathrm{PCa}$ (CRPC). Most CRPC cases continue, at least initially, to rely on AR signaling. The means by which AR drives CRPC are incompletely characterized, but it is believed that mechanisms enable AR transactivation under low androgen conditions (10).

Targeting ER in BCa. According to the American Cancer Society, $\mathrm{BCa}$ is the second most common cancer among American women. An estimated 252,710 women will be newly diagnosed with BCa in 2017, and around 40,610 women will die from the disease (2). Primary treatment options for localized disease include surgery and radiation. Because approximately $75 \%$ of $\mathrm{BCa}$ expresses ER, inhibiting ER function is the goal of endocrine therapy; this is effective both in the adjuvant setting after surgery to reduce the risk of relapse and in patients with metastatic disease to slow disease progression (11).

Examples of endocrine therapy drugs include the selective ER modulator tamoxifen, which antagonizes ER in BCa while preserving its activating and estrogen-like functions in the bone (12). The full antagonist fulvestrant leads to ER degradation, while aromatase inhibitors reduce overall estrogen levels by preventing the conversion of androgens to estrogens $(13,14)$. The widespread application of these drugs as adjuvant therapies has led to a significant reduction in BCa mortality (15). However, not all ER-positive BCa patients respond to endocrine treatments and nearly all women with advanced cancer will eventually die from metastatic disease (16). As with PCa, it is thought that many endocrine therapyresistant breast tumors continue to rely on active ER signaling, 
A SR

\begin{tabular}{l|l|l|l} 
AF-1 & DBD & Hinge & AF-2 (LBD)
\end{tabular}
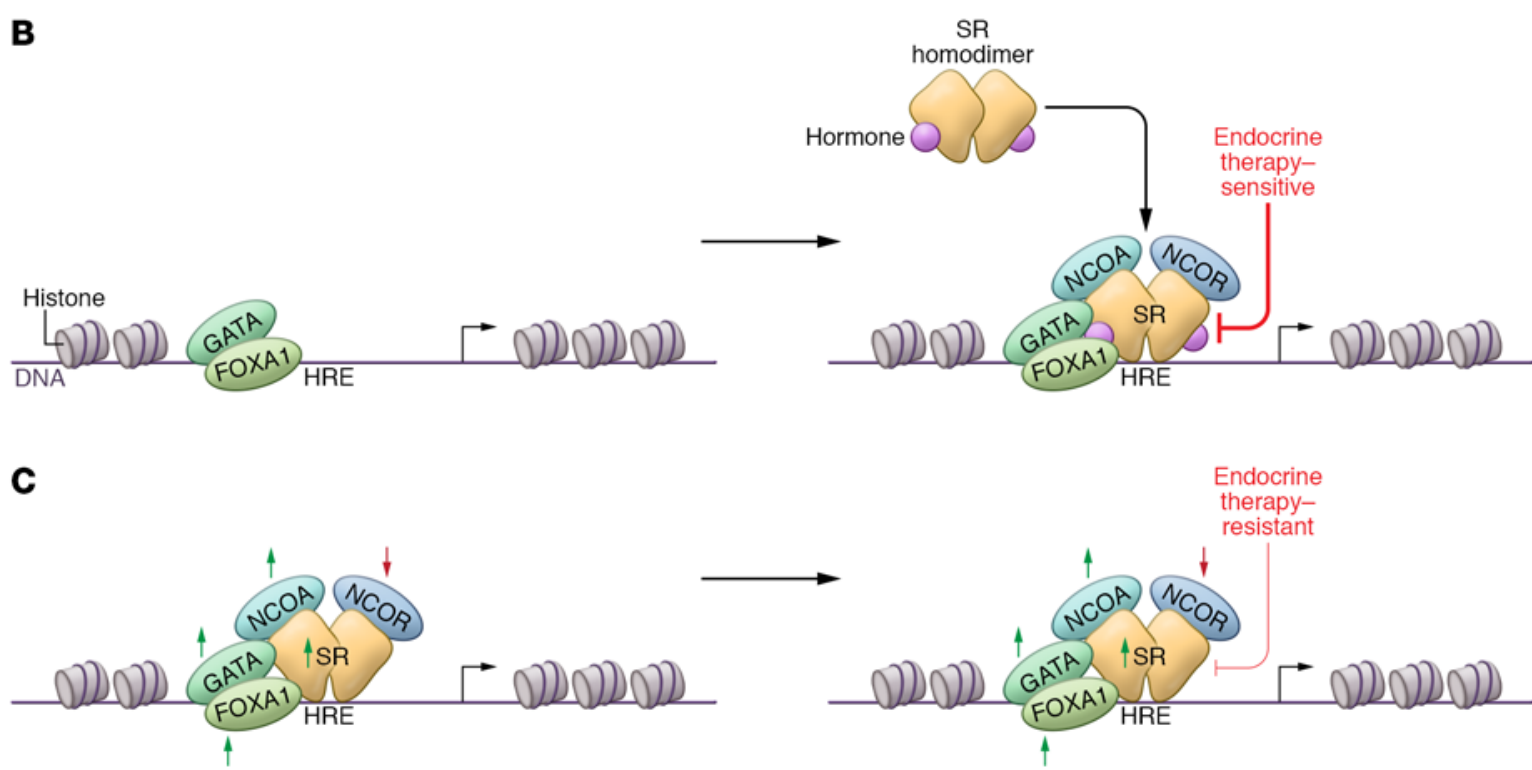

Figure 1. Steroid receptor (SR) structure and function in endocrine therapy-sensitive and -resistant cancer. (A) Schematic structure of the SR proteins $A R$ and $E R$, which belong to the nuclear receptor TF superfamily and harbor two transcriptional activation domains, the $\mathrm{N}$-terminal ligand-independent activation function domain (AF-1) and the C-terminal ligand-dependent AF-2 domain. The LBD also resides in the C terminus, while the DNA-binding domains (DBDs) and hinge domains are in the central core of the proteins. (B) SRs are the main targets of endocrine therapy, which induces tumor regression in sensitive PCa and BCa. In general, AR and ER function as ligand-dependent TFs that act as homodimers when activated in response to hormone binding. Androgens bind to $A R$, while estrogens bind to $E R$, and the respective receptor-ligand hormone complexes directly recognize specific DNA sequences harboring hormone response elements (HREs). Upon their DNA binding, transcriptional coregulators, including NCOAs and NCORs that mediate the regulation of hormone-responsive genes, are recruited. In addition, collaborating pioneer TFs of the FOXA1 and GATA families ensure the establishment of oncogenic gene expression programs that drive prostate and breast tumors. (C) Reactivation of SR in endocrine therapy-resistant PCa and BCa can be due to genetic alterations in the SR, in their transcriptional coregulators and in their pioneer factors, resulting in altered transcriptional activity. Enhanced activity in therapy-resistant disease is shown with green arrows pointing upward, whereas reduced activity is shown with red arrows pointing downward.

where ER transactivation is mediated by alternative, hormoneindependent mechanisms (17).

Endocrine therapy resistance and genomic hormone action. Despite the effectiveness of endocrine therapies in $\mathrm{PCa}$ and $\mathrm{BCa}$, intrinsic and acquired resistance remain a clinical challenge. Furthermore, both $\mathrm{PCa}$ and $\mathrm{BCa}$ are heterogeneous diseases with different subtypes that do not all respond to treatments in the same way. BCa patients can be stratified according to gene expression, which is predictive of the clinical course of disease; this is exemplified by ER-positive luminal subtypes that respond well to endocrine treatment $(18,19)$. In contrast, PCa subtypes are not well defined, and prognostic stratification of intermediate-risk patients based on gene expression profiles is still a challenge (20).

AR and ER share a common modular structure (Figure 1A) and play critical roles in the normal development and function of the prostate and breast, only becoming oncogenes in specific genetic contexts (21). They function as ligand-dependent TFs that bind to steroid hormones with their ligand-binding domain (LBD) and, upon DNA binding, regulate hormone-responsive transcription (Figure 1B). This transcriptional axis is altered during endocrine therapy resistance and functions despite low levels of activating hormone (Figure 1C).

\section{Proposed mechanisms leading to endocrine therapy resistance}

Many mechanisms contributing to endocrine therapy resistance in $\mathrm{PCa}$ and $\mathrm{BCa}$ have been proposed. It has been suggested that resistance to anti-hormone therapy is promoted by the presence of a preexisting hormone-independent tumor-initiating cell $(22,23)$ or by cellular plasticity that allows conversion to the hormone-independent phenotype. In this situation, hormone independence may be achieved by losing dependence on the steroid hormone receptordriven and lineage-defining gene expression programs. Consistent with this idea, it is estimated that $10 \%$ of patients with late-stage CRPC (24), and $15 \%$ to $20 \%$ of patients with ER-positive primary BCa that becomes metastatic lose receptor expression (25). Additionally, the dependency on a specific steroid receptor for gene regulation can be bypassed through the activity of alternative TFs. For example, in $\mathrm{PCa}$, administration and ensuing resistance to AR-targeted therapies coincides with the upregulation of the glucocorticoid receptor (GR) (26-28). GR induction is further associated with restored expression of a subset of AR target genes implicated in mediating enzalutamide resistance in xenografts (28). However, in the majority of endocrine therapy-resistant cases the relevant steroid receptors remain active and are crucial for tumor proliferation and survival $(10,17)$. 
Table 1. Genetic alterations in the transcriptional AR/ER axis in primary and endocrine therapy-resistant PCa and BCa

\begin{tabular}{|c|c|c|c|c|c|c|c|}
\hline \multirow[t]{2}{*}{ Molecule } & \multirow{2}{*}{$\begin{array}{l}\text { Levels in endocrine } \\
\text { therapy resistance }\end{array}$} & \multirow[t]{2}{*}{ Stage } & \multicolumn{3}{|c|}{ Frequency of alterations } & \multirow{2}{*}{$\begin{array}{c}\text { Total frequency } \\
\text { of alterations }\end{array}$} & \multirow[t]{2}{*}{ Reference } \\
\hline & & & Amplification & Deletion & Point mutation & & \\
\hline \multicolumn{8}{|c|}{ PCa genetic alterations } \\
\hline$A R$ & & CRPC & $52 \%$ & - & $18 \%$ & $62.7 \%^{\mathrm{A}}$ & 38 \\
\hline \multirow[t]{2}{*}{ NCOR1/2 } & Downregulated & Primary PCa & $0.9 \%$ & $3.9 \%$ & $1.8 \%$ & $6.3 \%$ & 46 \\
\hline & & CRPC & - & $1.3 \%$ & $6.7 \%$ & $12.7 \%^{\mathrm{B}}$ & 38 \\
\hline \multirow[t]{2}{*}{ FOXA1 } & Upregulated & Primary PCa & $2.1 \%$ & $0.6 \%$ & $3.9 \%$ & $6.3 \%$ & 46 \\
\hline & & CRPC & $4.7 \%$ & - & $10.7 \%$ & $15.3 \%$ & 38 \\
\hline \multirow[t]{2}{*}{ GATA2 } & Upregulated & Primary PCa & $3.3 \%$ & $0.3 \%$ & - & $3.6 \%$ & 46 \\
\hline & & CRPC & $6.7 \%$ & - & - & $6.7 \%$ & 38 \\
\hline \multicolumn{8}{|c|}{$\mathrm{BCa}$ genetic alterations } \\
\hline \multirow[t]{2}{*}{ FOXA1 } & Upregulated & Primary BCa & $1.7 \%$ & - & $1.7 \%$ & $3.3 \%{ }^{\mathrm{C}}$ & 71 \\
\hline & & Primary BCa, ILC & $1.8 \%$ & - & $7 \%$ & $8.8 \%$ & 112 \\
\hline GATA3 & Possibly upregulated & Primary BCa & $2.5 \%$ & - & $10.4 \%$ & $12.9 \%$ & 71 \\
\hline
\end{tabular}

-, none; n.a.: not available. ${ }^{A} A R$ rearrangements are detected in $30 \%$ of men with CRPC (41). ${ }^{B} N C O R 1 / 2$ fusions are detected in an additional $4.7 \%$ of men with CRPC (38). ' ${ }^{C}$ OXA1 copy number gains are detected in $20 \%$ of people with primary $\mathrm{BCa}(71,104)$.

The reactivation of AR- and ER-dependent transcription during endocrine therapy resistance can occur through two major mechanisms. First, the steroid hormone genomic signaling axis itself can adapt to endocrine therapy by augmenting its activity and its responsiveness to hormones, mainly through genomic alterations (discussed in detail below). Second, the activated kinase cascades that mediate growth factor receptor-dependent signaling frequently exhibit increased activity during cancer progression and can mediate phosphorylation of AR, ER, and their transcriptional coregulators, thereby contributing to hormoneindependent activation of gene expression (29-31). These steroid receptor transactivation mechanisms are not mutually exclusive, but instead result in different gene expression programs (32). For example, in BCa cells, EGF induces ER binding sites across the genome ("cistromes") that are distinct from those induced by estrogen (33). ER at EGF-induced cistromes shows corecruitment and dependence on the TF AP-1; this differs from ER at estrogen-induced sites, suggesting a change in collaborating factors under different activation conditions (33). Similarly, in men with CRPC, AP-1 binding motifs are overrepresented at AR binding sites located at predicted CRPC drivers (34). Ultimately, the improved characterization of growth factor- and hormone-dependent steroid receptor activation and how they cross-talk should provide the basis for improving endocrine therapy.

\section{Restoration of the transcriptional AR/ER signaling axis}

In endocrine therapy-resistant cancers, reactivation of steroid receptor signaling can emerge as a consequence of genetic alterations in the AR/ER-dependent transcriptional signaling axis.
Endocrine therapy generally can be considered a constraint that selects for specific genetic factors that enhance hormone signaling, ultimately providing a growth advantage for the tumor cells that harbor the alteration. This idea is the basis for the clonal evolution model that has been proposed to act in progressing $\mathrm{PCa}$ and $\mathrm{BCa}(35,36)$. Indeed, both types of malignancy show an increase in mutations along the AR and ER signaling axes upon endocrine treatment, suggesting that these genetic events are selected for under the pressure of endocrine treatment. Below we discuss the molecular effects of these genetic determinants on AR- and ER-dependent transcription during the emergence of endocrine therapy resistance.

\section{Genomic alterations of steroid receptors}

Steroid receptor amplifications. In men with $\mathrm{PCa}, A R$ amplification was first observed in 7 of 23 (30.4\%) tumors that recurred under ADT; this genetic alteration was absent in matched primary tumors prior to treatment (37). The rate of $A R$ amplification was even higher (52\%) in a more recent study (Table 1 and ref. 38). In $\mathrm{BCa}$, the clinical significance of ESR1 gene amplifications in driving recurrent disease is less clear, since recent studies showed an amplification rate of around $2 \%$ in both primary and metastatic disease (Table 1 and ref. 36). Overexpression of AR and ER allows tumor growth under hormone-depleted conditions in models of PCa and BCa $(39,40)$. Therefore, amplifications that result in increased levels of steroid receptors may be functional drivers of proliferation in an endocrine therapy-resistant setting.

Steroid receptor splice variants. A recent study revealed a positive correlation between $A R$ copy number increase and upregulation of LBD-deficient AR splice variants (AR-Vs) (41). The find- 
ings further suggested that $A R$ genomic rearrangements enhance the production of AR-Vs in a subset of patients (41). AR-Vs can promote resistance by engaging AR chromatin-binding sites (42) and by driving AR-dependent transcription in a constitutive ligand-independent manner (43-45). Notably, AR-Vs can arise in the absence of genetic alterations and are detected in normal prostate tissue $(44,46)$, where they may contribute to endogenous AR signaling. Consistent with AR-V levels being correlated with progression, detection of the AR-V ARv7 in circulating tumor cells from abiraterone- or enzalutamide-treated patients with CRPC is associated with therapeutic resistance and decreased overall survival rates $(47,48)$.

The clinical implication of ER splice variants in recurrent $\mathrm{BCa}$ is less well established than implications of AR-Vs. It is possible that ER splice variants are less potent transcriptional activators than AR-Vs, as the N-terminal AF-1 domain of ER is a weaker transcriptional activator than the corresponding AR domain (49) and may be unable to activate target genes in a hormone-independent manner. In support of this idea, a YAP1-ESR1 translocation has been identified in a metastatic ER-positive BCa (40). In this fusion, the AF-1 domain, the DNA-binding domain, and the hinge region of ER are joined to regions of the YAP1 protein that contain a transactivation domain. Expression of this chimeric protein promotes ligand-independent tumor growth and resistance to fulvestrant (40), reminiscent of the effect of AR-Vs in PCa. Future studies will reveal the frequency and functional impact of such events in endocrine therapy-resistant disease.

Steroid receptor point mutations. More than 20 years ago, AR mutations were detected in men with CRPC, but not in patients with primary disease, supporting the idea of continued AR dependence in these cases $(50,51)$. More recently the AR LBD has emerged as a mutational hotspot with four major missense mutations (L702H, W742C, H875Y, and T878A) that are found in $15 \%$ to $20 \%$ of CRPC cases (Table 1 and refs. 38,52 ). Characterization of AR T878A, H875Y, and W742C showed that the mutants were stimulated rather than inhibited by the AR antagonists nilutamide, flutamide, and bicalutamide, respectively (53-55). This antagonist-to-agonist switch results in a dependency of AR transactivation on these agents and is consistent with the "anti-androgen withdrawal response" that is often seen in PCa patients upon treatment termination (56). Interestingly, antagonist-to-agonist conversion is not only seen in the presence of AR point mutants but also in AR-overexpressing cells (39). Therefore, this clinical manifestation may also be linked to an amplification-dependent increase in AR levels. Another proposed mode of action for AR mutations is enhanced sensitivity to an increased spectrum of agonists, which allows for transcriptional activation by noncanonical steroid ligands, including adrenal androgens, estrogen, progesterone, and glucocorticoids (57-59). Although there is no direct clinical evidence that these alternative ligands drive CRPC - and although metabolic conversion of the ligands to testosterone or dihydrotestosterone may occur - the clinical observation that not all patients harboring specific antagonist-to-agonist switch mutations were treated with the respective antagonist $(38,60)$ is consistent with the idea that an increased ligand repertoire or increased ligand sensitivity is important for AR mutant-driven CRPC.
Studies in the 1990s identified potential endocrine resistance mutations in a small number of BCa patients (61), and studies in cell lines indicated that the identified LBD mutations confer gain of function, resulting in both ligand-independent and enhanced ligand-stimulated ER transcriptional activity $(62,63)$. More recently, five studies identified missense point mutations in the ER LBD in metastatic BCa. Overall, 187 metastatic ER-positive BCa samples from patients undergoing endocrine treatment were sequenced in these studies, and ER LBD mutations were identified in 39 patients (21\%) (Table 1 and refs. 36, 40, 64-66). Jeselsohn et al. found a correlation between the prevalence of hotspot ER LBD mutations and the number of lines of endocrine treatment (36). The most common missense mutations were D538G and those affecting the $\mathrm{Y} 537$ residue, which showed amino acid changes to S, $\mathrm{N}$, and $\mathrm{C}$. Several other mutations in the LBD that can confer resistance have been found. Consistent with an expansion of the ER transcriptional network being responsible for this phenotype, gene expression profiling showed that mutant ER activates both known estrogen-induced genes and novel targets. Importantly, fulvestrant and tamoxifen both blocked mutant ER, although the required doses were substantially higher than for WT ER (36, 40, 64-66).

The LBD of all steroid receptors is folded into $12 \alpha$-helices, where helices 3, 4, and 12 are integral for the structural response of ligand binding, ultimately leading to recruitment of transcriptional coactivators to the AF-2 domain. Structural studies comparing WT and Y537S ER proteins revealed that in the absence of ligand, helix 12 in mutant ER is stabilized in the agonistic conformation, similar to that of estrogen-bound WT ER. Consistently, multiple ER coactivators were recruited to the ER mutant AF-2 in a ligandindependent manner. In PCa patients with AR gain-of-function LBD mutations, AR is transcriptionally active because mutations such as T878A and W742L result in an antagonist-bound activating conformation, where helix 12 is in the agonistic state (67). Promiscuous activity of ligands, such as glucocorticoids on the $\mathrm{L} 702 \mathrm{H}$ mutation, may be explained structurally by the fact that residues within the ligand-binding pocket not only determine the structure of the LBD, but also dictate the types of ligands that can be accommodated (68).

In general, the presence of these gain-of-function AR and ER mutations in tumors requires the development of better antagonists for endocrine treatment. Ideally, these molecules should bind more strongly than hormone to the LBD without permitting coactivator binding to AF-2. This would result in an unstable receptor conformation, leading to its degradation, as exemplified by fulvestrant (14).

\section{Alterations in collaborating factors}

Transcriptional coregulators. Gene regulation by steroid receptors requires positive and negative transcriptional coregulators, termed nuclear receptor corepressors (NCORs) and nuclear receptor coactivators (NCOAs). The enzymatic activity associated with these coregulator complexes leads to decreases or increases in acetylation of local chromatin. Because acetylation relaxes chromatin, increased transcriptional activity is observed at hyperacetylated loci, while decreased activity is seen at hypoacetylated regions. Acetylation is mediated by p300 histone acetyltransferase enzymes, which associate with the p160 family of NCOAs (NCOA1-3). Removal of acetyl groups from chromatin counteracts 
activation and prevents transcription. This reaction is catalyzed by histone deacetylases such as HDAC3, which interact with NCOR1and NCOR2-containing protein complexes $(69,70)$.

NCOR. In PCa, the frequency of potential loss-of-function alterations in NCOR $1 / 2$ increases from around $6 \%$ in primary disease to $13 \%$ in metastatic CRPC (Table 1 and refs. 38, 46). Similarly, in BCa NCOR1/2 show putative inactivating mutations in approximately $6 \%$ of primary tumors (Table 1 and ref. 71 ). Tamoxifen mediates its inhibitory effect on estrogen-responsive genes by locking the ER LBD into a conformation that prevents recruitment of NCOA1-3 and promotes the recruitment of NCOR1/2. Tamoxifen resistance has been associated with downregulation of NCOR1 expression (72), and loss of NCOR1 expression is sufficient to trigger resistance in experimental models (73). Thus, it is possible that inactivating NCOR1/2 mutations may also contribute to the emergence of tamoxifen resistance.

NCOA. NCOA2 amplification is seen in $6 \%$ of PCa patients with primary disease and in $16 \%$ with metastasis (Table 1 and refs. $38,46)$, and an increased NCOA2 level was associated with the development of CRPC (74). Clinical and experimental approaches have demonstrated that NCOA2 is an important regulator of endocrine response in PCa. It is thought that the clinical AR antagonist bicalutamide functions by decreasing coactivator recruitment and that enhanced coactivator activity may contribute to resistance (75). NCOA2 depletion reduces AR-dependent gene expression in PCa cells (74). Moreover, in a genetically engineered mouse model, overexpression of NCOA2 in the prostate epithelium drives neoplasia and promotes metastasis through hyperactivation of growth factor signaling (76). Therefore, NCOA2 contributes to endocrine therapy-resistant phenotypes by promoting AR-dependent and -independent mechanisms.

In $\mathrm{BCa}, \mathrm{NCOA} 2$ and $\mathrm{NCOA} 3$ are amplified in $8 \%$ of primary tumors (Table 1 and ref. 71). In the mouse mammary gland, NCOA3 overexpression contributed to mammary tumor development via estrogen-dependent and -independent mechanisms (77, 78). In patients, elevated NCOA3 expression is associated with a higher risk of developing tamoxifen resistance (79). Additionally, because NCOA3 can be activated by growth factor-mediated phosphorylation (30), patients who express high levels of both NCOA3 and the receptor tyrosine kinase HER2 have an even greater chance of developing tamoxifen resistance (79). Functionally, elevated NCOA3 expression increases the agonistic activities of tamoxifen-bound ER, thereby reducing its anti-tumor activity (80).

Reinstating NCOR activity in endocrine therapy-resistant PCa and BCa will be challenging. Paradoxically, a recent clinical study demonstrated that inhibitors of HDAC activity can resensitize endocrine therapy-resistant BCa to tamoxifen (81). The molecular mechanisms underlying this phenomenon are only starting to be understood (82) but are likely to involve NCOR-independent processes. Therapeutic interference with the augmented dependence on specific coactivators may also be feasible, as evidenced by the development of NCOA1/3-specific tool compound inhibitors (83, 84). Therefore, it is conceivable that relapsed patients harboring NCOA gene amplifications and/or overexpression may benefit from NCOA inhibitors.

Pioneer TFs: FOXA1, GATA2, and GATA3. Pioneer TFs interact with their recognition sequences in condensed chromatin before activating transcription, and they have the capacity to increase the accessibility of local chromatin structures, allowing productive binding of other tissue-specific TFs, such as steroid receptors. Thus, pioneer TFs are integral for establishing cell lineage identity (85). FOXA1 as well as GATA family members are the pioneer TFs that facilitate genomic binding of AR and ER, and they are determinants of mammary and prostate epithelial lineages $(86,87)$. In PCa cells, FOXA1 and GATA2 cobind with AR, while in BCa cells, FOXA1, GATA3, and ER colocalize to the genome. Binding of these multicomponent TF complexes mediates activation of luminal epithelium- and cancer-associated gene expression programs (88-92). Since pioneer TF binding to the genome occurs in a hormoneindependent manner, these TFs potentially have an intrinsic capacity to drive steroid receptor-dependent transcription in endocrine therapy-resistant cancer cells. Below, we explore this idea in light of recent findings of genetic alterations in pioneer TFs.

FOXA1. Cistrome analyses for AR, ER, and FOXA1 in PCa and BCa cell lines revealed cooccupancy between FOXA1 and the steroid receptors $(88,89,93)$. Moreover, the observed coexpression of FOXA1 with AR in PCa and with ER in BCa is consistent with FOXA1 playing an important role in shaping AR- and ER-dependent transcriptional programs in these cancers $(94,95)$. In PCa patients, high levels of FOXA1 correlate with a shorter time to recurrence $(96,97)$. Increased FOXA1 expression is also observed in most metastatic and CRPC cases $(95,96)$. Conversely, in BCa, FOXA1 levels are associated with a good prognosis in ER-positive BCa patients $(94,98)$, whereas high levels of both ER and FOXA1 are expressed in endocrine therapy-resistant metastases (99). The discrepancy of FOXA1 being a "good" or "bad" factor in primary $\mathrm{PCa}$ or $\mathrm{BCa}$, respectively, can be explained by distinct modes of chromatin targeting, resulting in different dependencies of AR and ER on FOXA1. Specifically, ER requires FOXA1 to bind chromatin, whereas AR can bind in its absence (100-102). This fundamental difference helps explain why FOXA1 is a positive predictor for endocrine therapy response in $\mathrm{BCa}$, since its presence mediates a functional ligand-dependent ER transcriptional complex that is sensitive to ER antagonism. In PCa, the AR cistrome is FOXA1 independent; therefore, FOXA1 is not a marker for endocrine therapy response but instead may promote the acquisition of novel, potentially oncogenic binding sites.

In endocrine therapy-resistant disease, both $\mathrm{PCa}$ and $\mathrm{BCa}$ patients have elevated FOXA1 levels, consistent with FOXA1 being a driver of the ligand-independent phenotype $(95,96,99)$. Furthermore, ER cistrome mapping in patients with BCa suggested that FOXA1-dependent reprogramming of ER sites correlated with worse clinical outcome (99). The oncogenic role of FOXA1 may also be linked to its genetic alteration, the functional effects of which are discussed below.

FOXA1 amplifications. In PCa, the frequency of FOXA1 amplification increases from $2 \%$ in primary disease to $5 \%$ in CRPC (Table 1 and refs. 38, 46). In PCa xenograft models, larger tumors are induced upon FOXA1 overexpression, suggesting that FOXA1 amplification may have functional consequences in clinical disease (52). In PCa cells, FOXA1 overexpression increases proliferation rates under low-androgen conditions (103). This phenotype is linked to the FOXA1-dependent acquisition of new AR genomic binding sites that activate a novel, CRPC-like transcriptional profile 
that is enriched in oncogenic signaling pathways known to promote cell growth and survival in a hormone-starved environment (103).

FOXA1 amplification and copy number gain are seen in $20 \%$ of primary BCa (Table 1 and refs. 71, 104). Further, FOXA1 copy number is significantly increased in lymph node metastases compared with primary matched ER-positive tumors (104) and is increased in patients receiving endocrine treatment (105). Similar to PCa, overexpression of FOXA1 in ER-positive BCa cell lines promotes resistance to tamoxifen and to estrogen deprivation (104), and gene expression profiling revealed stimulation of oncogenic signaling pathways that promote ligand-independent ER activation.

Taken together, these results suggest a model in which increased FOXA1 levels reprogram and adapt AR- and ER-dependent transcription in response to endocrine therapy. FOXA1 likely acts in two ways: first, FOXA1 induces signaling-dependent phosphorylation and activation of AR and ER by promoting expression of genes in growth factor receptor-driven pathways. Second, FOXA1 creates novel genomic binding sites for the ligandindependent steroid receptors. The potential interplay between FOXA1, steroid hormone signaling, and growth factor receptor pathways provides opportunities for targeted therapeutic strategies in the context of endocrine therapy failure. For example, the concerted upregulation of IL-8 by FOXA1 and ER in endocrine therapy-resistant $\mathrm{BCa}$ cells identifies a potential target for the treatment of ER-positive, FOXA1-high patients (104). Moreover, FOXA1 also drives proliferation in PCa and BCa cell lines (100, 106), suggesting that it can also be considered as a therapeutic target. It is possible that compounds selectively targeting FOXA1 may be identified since the transcriptional activity of a related factor, FOXM1, can be inhibited by a small molecule (107).

FOXA1 point mutations. Several whole exome sequencing studies have revealed that FOXA1 harbors recurrent mutations in $4 \%$ of patients with primary PCa and $11 \%$ with CRPC (Table 1 and refs. $38,46,108)$. Two mutational hotspots in or around the DNA-binding Forkhead domain and the C-terminal transactivation domain were identified. The most common mutations in primary tumors affect the Forkhead domain and are predicted to change FOXA1 DNA binding (46). Gene expression studies comparing mutated FOXA1-carrying with WT-carrying primary tumors revealed increased levels of AR target gene activity in the former (46), which is in agreement with the hypothesis that mutant, more than WT FOXA1, promotes AR signaling and favors a more oncogenic AR-dependent transcriptional program that drives PCa progression. Because FOXA1 appears to contribute to reprogramming of the AR cistrome and downstream gene expression in primary PCa tumors (109) and in CRPC (110), the impact of FOXA1 mutations on this process should be determined. A fraction of the FOXA1 mutations would potentially give rise to loss-of-function truncated proteins. Therefore, FOXA1 inactivation may be an additional mechanism that influences AR-dependent transcription. In agreement with this, it has been proposed that decreased levels of FOXA1 significantly alter the AR cistrome (111).

The Cancer Genome Atlas (TCGA) initiative performed largescale genome sequencing of different cancer types, including $\mathrm{BCa}$. In their initial report of this study, FOXA1 point mutations were found in 8 of 482 tumors (1.7\%), all of which were ER positive
(Table 1 and ref. 71). More recently, specific profiling of invasive ER-positive lobular BCa (ILC), the second most prevalent subtype after invasive ductal carcinoma (IDC), revealed that $7 \%$ of ILC harbored FOXA1 mutations (Table 1 and ref. 112). As in PCa, these mutations affected the Forkhead domain and the C-terminal transactivation domain (112), suggesting that these regional FOXA1 hotspot mutations are tissue independent and may contribute to oncogenic transcriptional programs in $\mathrm{PCa}$ and $\mathrm{BCa}$ in an as yet unknown manner. Although some FOXA1 mutations were predicted to give rise to truncated proteins, an indirect measurement of FOXA1 activity in this dataset revealed no decrease (112), suggesting that overall FOXA1 mutations are active. Gene expression profiling comparing FOXA1-mutant and WT ILC tumors showed increased expression of neuroendocrine lineage genes in FOXA1 mutant tumors, which is consistent with mutant FOXA1 reprogramming the cell type-specific ER transcriptional program (112). The precise impact of mutant FOXA1 as well as its interplay with WT FOXA1 have not been thoroughly investigated, and further studies are needed to develop potential FOXA1 mutant-targeting strategies.

GATA2 in PCa. Another AR-interacting pioneer is GATA2. Cistrome analyses in PCa cells revealed that AR binding sites were significantly enriched in GATA motifs (89). Moreover, GATA2 was shown to play an essential role in AR-chromatin interactions, resulting in androgen-dependent gene activation $(113,114)$. There is also a positive correlation between GATA2 and AR expression, reflecting the direct activation of $A R$ by $\operatorname{GATA} 2(113,115)$. Additionally, GATA2 and AR cooperatively regulate androgen-dependent genes in high-risk PCa patients, where GATA2 is overexpressed and correlates with a more aggressive phenotype (116) and a higher risk of disease recurrence (117).

GATA2 point mutations are rarely detected (52), whereas gene amplification was seen in 3\% of patients with PCa and $7 \%$ with CRPC (Table 1 and refs. 38, 46). However, the functional consequences of these genetic alterations have not been studied. GATA2 has been implicated in activating IGF2 expression (118), which has been proposed to contribute to PCa progression in cellular models and in patients (119). IGF signaling is also thought to mediate chemotherapy resistance in PCa (118), suggesting that the IGF axis provides a therapeutic opportunity in patients with enhanced GATA2 function.

GATA3 in BCa. GATA3 is an ER-interacting pioneer TF that is required for estrogen-dependent cell cycle progression in ER-positive BCa cells (120). Cistrome data revealed that GATA3 enhances ER genome access (121). Moreover, GATA3 engages in a regulatory feed-forward loop with ER, whereby GATA3 increases ER expression and ER increases GATA3 levels (120). Accordingly, GATA3 and ER levels strongly correlate in BCa patients, and several studies have indicated that GATA3 is a predictor of endocrine therapy response (122). This clinical observation is consistent with the idea that luminal lineage determinants, including ER, FOXA1, and GATA3, are indicators for a functional ER-dependent transcriptional complex that is responsive to endocrine therapy.

GATA3 mutations occur at a high frequency $(10.4 \%)$ in primary $\mathrm{BCa}$ (Table 1 and refs. 71, 123-126) and are mutually exclusive with FOXA1 mutations $(71,112)$. Moreover, FOXA1 mutations are more prevalent in ILC (7\%), whereas GATA3 mutations are higher 
in IDC. FOXA1 and GATA3 are both key regulators of ER activity, suggesting that IDC and ILC may rely on different mechanisms to mediate their ER transcriptional programs.

The functional consequences of GATA3 mutations are only starting to be determined. Of the 54 mutations described, 49 result in frameshifts, which give rise to either truncated or C-terminally extended GATA3 proteins (GATA3-ext) (71). Frameshift mutations generally are believed to yield inactive proteins; however, recent findings suggest that this is not the case with GATA3 $(127,128)$. The proposed gain-of-function activity of mutant GATA3 may be linked to its ability to dimerize with WT GATA3 (129). Accordingly, it was shown that truncated GATA3, while showing decreased DNA binding activity, stabilized GATA3 WT/ mutant heterodimers (128). This finding may have important repercussions since GATA3 affects ER regulation both by activating ESR1 transcription and by acting as a collaborating factor for ER-dependent transcription $(120,121)$. Therefore, increases in GATA3-ext stability may alter the kinetics of this transcriptional network, resulting in potentially novel oncogenic functions.

A study of C-terminally extended GATA3 mutants also suggested gain-of-function activity (127). Reanalyses of the TCGA $\mathrm{BCa}$ patient cohort revealed that the specific frameshifts that give rise to GATA3-ext proteins are under positive selective pressure in patients (127). In contrast to the behavior of other GATA3 mutations in the same cohort, GATA3-ext was associated with reduced disease-free survival, suggesting that these tumors display a different pathology with respect to recurrence. However, the prognostic value of GATA3 mutations is still a matter of debate. Improved disease-free and overall survival were significantly correlated with GATA3 mutations in one study (130), whereas only marginal significance was seen for improved overall survival in ER-positive patients in the TCGA and METABRIC cohorts $(130,131)$. Therefore, not only the functional role of GATA3 mutations but also the interpretation of their clinical role requires further study.

\section{Conclusions and outlook}

Acquired resistance to various targeted therapies, including endocrine approaches, can be due to secondary genetic aberrations that alter the target protein, additional components of its pathway, or other compensatory pathways, thereby counteracting the inhibitory effect of the drug. Herein we addressed different mechanisms in light of the role of coding mutations and their effects on steroid receptor genomic activity in endocrine therapy-resistant PCa and BCa. We should mention, however, that alterations in noncoding genomic sequences are also gaining in importance and changes in cis-regulatory elements recognized by steroid receptors during cancer progression are a crucial level of transcriptional control. In this context, AR and ER bind to enhancer sequences, which enable the precise regulation of gene expression while acting over large distances from their target genes through physical interactions. The accessibility of enhancers can be affected by epigenetic alterations as well as by genetic mutations. For example, allele-specific recruitment of AR and AP-1 account for the increased enhancer activity that drives upregulation of oncogenic SOX9 in men harboring the 17q24.3 PCa risk locus (132). Additionally, the BCa risk locus 16q12.1 is thought to alter FOXA1 enhancer recruitment, resulting in the downregulation of the TOX3 tumor suppressor gene (133).

In the future, the molecular understanding of the role of specific genetic and epigenetic factors such as EZH2 (134-136) will provide the basis for tailoring cancer treatments through the course of disease for people with $\mathrm{PCa}$ and $\mathrm{BCa}$. Thus, the complex interplay between FOX, GATA, and steroid receptor-dependent gene regulation in cancer cells, which is influenced by the amount of the proteins, their mutational state, and their cistromes, needs to be more precisely described.

\section{Acknowledgments}

We acknowledge Nancy E. Hynes and Patrick Tschopp for discussion and help with preparing the manuscript. We apologize to all the colleagues whose work could not be cited due to space limitations. This work was supported by NIH grant 5P01 CA163227 (to $\mathrm{MB}$ ) and by an Ambizione grant PZOOP3_167938 from the Swiss National Science Foundations (to ACG).

Address correspondence to: Myles Brown, 450 Brookline Ave., Dana 730, Boston, Massachusetts 02215, USA. Phone: 617.632. 3948; E-mail: Myles_Brown@dfci.harvard.edu.
1. Jemal A, Bray F, Center MM, Ferlay J, Ward E, Forman D. Global cancer statistics. CA Cancer J Clin. 2011;61(2):69-90.

2. Siegel RL, Miller KD, Jemal A. Cancer statistics, 2017. CA Cancer J Clin. 2017;67(1):7-30.

3. Penney KL, et al. Gleason grade progression is uncommon. Cancer Res. 2013;73(16):5163-5168.

4. Siegel RL, Sahar L, Portier KM, Ward EM, Jemal A. Cancer death rates in US congressional districts. CA Cancer J Clin. 2015;65(5):339-344.

5. Huggins C, Hodges CV. Studies on Prostatic Cancer. I. The Effect of castration, of estrogen and of androgen injection on serum phosphatases in metastatic carcinoma of the prostate. Cancer Res. 1941;1(4):293-297.

6. van Poppel H, Nilsson S. Testosterone surge: rationale for gonadotropin-releasing hormone blockers? Urology. 2008;71(6):1001-1006.

7. Attard G, Belldegrun AS, de Bono JS. Selective blockade of androgenic steroid synthesis by novel lyase inhibitors as a therapeutic strategy for treating metastatic prostate cancer. BJU Int 2005;96(9):1241-1246.

8. de Bono JS, et al. Abiraterone and increased survival in metastatic prostate cancer. $N$ Engl JMed. 2011;364(21):1995-2005.

9. Tran C, et al. Development of a second-generation antiandrogen for treatment of advanced prostate cancer. Science. 2009;324(5928):787-790.

10. Watson PA, Arora VK, Sawyers CL. Emerging mechanisms of resistance to androgen receptor inhibitors in prostate cancer. Nat Rev Cancer. 2015;15(12):701-711.

11. Williams N, Harris LN. The renaissance of endocrine therapy in breast cancer. Curr Opin Obstet Gynecol. 2014;26(1):41-47.

12. Jaiyesimi IA, Buzdar AU, Decker DA, Hortobagyi GN. Use of tamoxifen for breast cancer: twenty- eight years later. J Clin Oncol. 1995;13(2):513-529. 13. Osborne CK. Aromatase inhibitors in relation to other forms of endocrine therapy for breast cancer. Endocr Relat Cancer. 1999;6(2):271-276.

14. Howell A, Osborne CK, Morris C, Wakeling AE. ICI 182,780 (Faslodex): development of a novel, “pure" antiestrogen. Cancer. 2000;89(4):817-825.

15. Baum M, et al. Anastrozole alone or in combination with tamoxifen versus tamoxifen alone for adjuvant treatment of postmenopausal women with early breast cancer: first results of the ATAC randomised trial. Lancet. 2002;359(9324):2131-2139.

16. Massarweh S, et al. Mechanisms of tumor regression and resistance to estrogen deprivation and fulvestrant in a model of estrogen receptor-positive, HER-2/neu-positive breast cancer. Cancer Res. 2006;66(16):8266-8273.

17. Osborne CK, Schiff R. Mechanisms of endocrine resistance in breast cancer. Annu Rev Med. 
2011;62:233-247.

18. Perou CM, et al. Molecular portraits of human breast tumours. Nature. 2000;406(6797):747-752.

19. Parker JS, et al. Supervised risk predictor of breast cancer based on intrinsic subtypes. JClin Oncol. 2009;27(8):1160-1167.

20. Yin Y, Zhang Q, Zhang H, He Y, Huang J. Molecular signature to risk-stratify prostate cancer of intermediate risk. Clin Cancer Res. 2017;23(1):6-8.

21. Garraway LA, Sellers WR. Lineage dependency and lineage-survival oncogenes in human cancer. Nat Rev Cancer. 2006;6(8):593-602.

22. Luo M, et al. Breast cancer stem cells: current advances and clinical implications. Methods Mol Biol. 2015;1293:1-49.

23. Shibata M, Shen MM. Stem cells in geneticallyengineered mouse models of prostate cancer. Endocr Relat Cancer. 2015;22(6):T199-T208.

24. Beltran H, et al. Divergent clonal evolution of castration-resistant neuroendocrine prostate cancer. Nat Med. 2016;22(3):298-305.

25. Hoefnagel LD, et al. Prognostic value of estrogen receptor $\alpha$ and progesterone receptor conversion in distant breast cancer metastases. Cancer. 2012;118(20):4929-4935.

26. Yemelyanov A, et al. Differential targeting of androgen and glucocorticoid receptors induces ER stress and apoptosis in prostate cancer cells: a novel therapeutic modality. Cell Cycle. 2012;11(2):395-406.

27. Isikbay M, et al. Glucocorticoid receptor activity contributes to resistance to androgen-targeted therapy in prostate cancer. Horm Cancer. 2014;5(2):72-89.

28. Arora VK, et al. Glucocorticoid receptor confers resistance to antiandrogens by bypassing androgen receptor blockade. Cell. 2013;155(6):1309-1322.

29. Kato S, et al. Activation of the estrogen receptor through phosphorylation by mitogen-activated protein kinase. Science. 1995;270(5241):1491-1494.

30. Font de Mora J, Brown M. AIB1 is a conduit for kinase-mediated growth factor signaling to the estrogen receptor. Mol Cell Biol. 2000;20(14):5041-5047.

31. Mellinghoff IK, Vivanco I, Kwon A, Tran C, Wongvipat J, Sawyers CL. HER2/neu kinasedependent modulation of androgen receptor function through effects on DNA binding and stability. Cancer Cell. 2004;6(5):517-527.

32. Dudek P, Picard D. Genomics of signaling crosstalk of estrogen receptor alpha in breast cancer cells. PLoS One. 2008;3(3):e1859.

33. Lupien M, et al. Growth factor stimulation induces a distinct ER(alpha) cistrome underlying breast cancer endocrine resistance. Genes Dev. 2010;24(19):2219-2227.

34. Sharma NL, et al. The androgen receptor induces a distinct transcriptional program in castrationresistant prostate cancer in man. Cancer Cell. 2013;23(1):35-47.

35. Gundem G, et al. The evolutionary history of lethal metastatic prostate cancer. Nature. 2015;520(7547):353-357.

36. Jeselsohn R, et al. Emergence of constitutively active estrogen receptor- $\alpha$ mutations in pretreated advanced estrogen receptor-positive breast cancer. Clin Cancer Res. 2014;20(7):1757-1767.

37. Visakorpi $\mathrm{T}$, et al. In vivo amplification of the androgen receptor gene and progression of human prostate cancer. Nat Genet. 1995;9(4):401-406.

38. Robinson D, et al. Integrative clinical genomics of advanced prostate cancer. Cell. 2015;161(5):1215-1228.

39. Chen CD, et al. Molecular determinants of resistance to antiandrogen therapy. Nat Med. 2004;10(1):33-39.

40. Li S, et al. Endocrine-therapy-resistant ESR1 variants revealed by genomic characterization of breast-cancer-derived xenografts. Cell Rep. 2013;4(6):1116-1130.

41. Henzler C, et al. Truncation and constitutive activation of the androgen receptor by diverse genomic rearrangements in prostate cancer. Nat Commun. 2016;7:13668

42. Chan SC, et al. Targeting chromatin binding regulation of constitutively active AR variants to overcome prostate cancer resistance to endocrine-based therapies. Nucleic Acids Res. 2015;43(12):5880-5897.

43. Dehm SM, Schmidt LJ, Heemers HV, Vessella RL, Tindall DJ. Splicing of a novel androgen receptor exon generates a constitutively active androgen receptor that mediates prostate cancer therapy resistance. Cancer Res. 2008;68(13):5469-5477.

44. Guo Z, et al. A novel androgen receptor splice variant is up-regulated during prostate cancer progression and promotes androgen depletion-resistant growth. Cancer Res. 2009;69(6):2305-2313.

45. Sun S, et al. Castration resistance in human prostate cancer is conferred by a frequently occurring androgen receptor splice variant. J Clin Invest. 2010;120(8):2715-2730

46. Cancer Genome Atlas Research Network. The molecular taxonomy of primary prostate cancer. Cell. 2015;163(4):1011-1025.

47. Antonarakis ES, et al. AR-V7 and resistance to enzalutamide and abiraterone in prostate cancer. N Engl J Med. 2014;371(11):1028-1038.

48. Antonarakis ES, et al. Androgen receptor splice variant 7 and efficacy of taxane chemotherapy in patients with metastatic castration-resistant prostate cancer. JAMA Oncol. 2015;1(5):582-591.

49. Lavery DN, McEwan IJ. Structure and function of steroid receptor AF1 transactivation domains: induction of active conformations. Biochem J. 2005;391(pt 3):449-464.

50. Taplin ME, et al. Mutation of the androgenreceptor gene in metastatic androgenindependent prostate cancer. $N$ Engl J Med. 1995;332(21):1393-1398.

51. Suzuki H, Sato N, Watabe Y, Masai M, Seino S, Shimazaki J. Androgen receptor gene mutations in human prostate cancer. J Steroid Biochem Mol Biol. 1993;46(6):759-765.

52. Grasso CS, et al. The mutational landscape of lethal castration-resistant prostate cancer. Nature. 2012;487(7406):239-243.

53. Veldscholte J, et al. A mutation in the ligand binding domain of the androgen receptor of human LNCaP cells affects steroid binding characteristics and response to anti-androgens. Biochem Biophys Res Commun. 1990;173(2):534-540.

54. Fenton MA, et al. Functional characterization of mutant androgen receptors from androgenindependent prostate cancer. Clin Cancer Res.
1997;3(8):1383-1388.

55. Yoshida T, et al. Antiandrogen bicalutamide promotes tumor growth in a novel androgen-dependent prostate cancer xenograft model derived from a bicalutamide-treated patient. Cancer Res. 2005;65(21):9611-9616.

56. Scher HI, Kelly WK. Flutamide withdrawal syndrome: its impact on clinical trials in hormonerefractory prostate cancer. J Clin Oncol. 1993;11(8):1566-1572

57. van de Wijngaart DJ, et al. Systematic structure-function analysis of androgen receptor Leu701 mutants explains the properties of the prostate cancer mutant L701H. J Biol Chem. 2010;285(7):5097-5105.

58. Zhao XY, et al. Glucocorticoids can promote androgen-independent growth of prostate cancer cells through a mutated androgen receptor. Nat Med. 2000;6(6):703-706.

59. Tan J, et al. Dehydroepiandrosterone activates mutant androgen receptors expressed in the androgen-dependent human prostate cancer xenograft CWR22 and LNCaP cells. Mol Endocrinol.1997;11(4):450-459.

60. Chen EJ, et al. Abiraterone treatment in castration-resistant prostate cancer selects for progesterone responsive mutant androgen receptors. Clin Cancer Res. 2015;21(6):1273-1280.

61. Zhang QX, Borg A, Wolf DM, Oesterreich S, Fuqua SA. An estrogen receptor mutant with strong hormone-independent activity from a metastatic breast cancer. Cancer Res. 1997;57(7):1244-1249.

62. Carlson KE, Choi I, Gee A, Katzenellenbogen BS, Katzenellenbogen JA. Altered ligand binding properties and enhanced stability of a constitutively active estrogen receptor: evidence that an open pocket conformation is required for ligand interaction. Biochemistry. 1997;36(48):14897-14905.

63. Lazennec G, Ediger TR, Petz LN, Nardulli AM, Katzenellenbogen BS. Mechanistic aspects of estrogen receptor activation probed with constitutively active estrogen receptors: correlations with DNA and coregulator interactions and receptor conformational changes. Mol Endocrinol.1997;11(9):1375-1386.

64. Toy W, et al. ESR1 ligand-binding domain mutations in hormone-resistant breast cancer. Nat Genet. 2013;45(12):1439-1445.

65. Robinson DR, et al. Activating ESR1 mutations in hormone-resistant metastatic breast cancer. Nat Genet. 2013;45(12):1446-1451.

66. Merenbakh-Lamin K, et al. D538G mutation in estrogen receptor- $\alpha$ : A novel mechanism for acquired endocrine resistance in breast cancer. Cancer Res. 2013;73(23):6856-6864

67. Estébanez-Perpiñá E, Jouravel N, Fletterick RJ. Perspectives on designs of antiandrogens for prostate cancer. Expert Opin Drug Discov. 2007;2(10):1341-1355.

68. Matias PM, et al. Structural basis for the glucocorticoid response in a mutant human androgen receptor (AR(ccr)) derived from an androgen-independent prostate cancer. J Med Chem. 2002;45(7):1439-1446.

69. Perissi V, Jepsen K, Glass CK, Rosenfeld MG Deconstructing repression: evolving models of co-repressor action. Nat Rev Genet. 
2010;11(2):109-123.

70. Xu J, Wu RC, O’Malley BW. Normal and cancerrelated functions of the $\mathrm{p} 160$ steroid receptor co-activator (SRC) family. Nat Rev Cancer. 2009;9(9):615-630.

71. Cancer Genome Atlas Network. Comprehensive molecular portraits of human breast tumours. Nature. 2012;490(7418):61-70.

72. Girault I, et al. Expression analysis of estrogen receptor alpha coregulators in breast carcinoma: evidence that NCOR1 expression is predictive of the response to tamoxifen. Clin Cancer Res. 2003;9(4):1259-1266.

73. Lu R, et al. COPS5 amplification and overexpression confers tamoxifen-resistance in ER $\alpha$-positive breast cancer by degradation of NCoR. Nat Commun. 2016;7:12044.

74. Agoulnik IU, et al. Androgens modulate expression of transcription intermediary factor 2 , an androgen receptor coactivator whose expression level correlates with early biochemical recurrence in prostate cancer. Cancer Res. 2006;66(21):10594-10602.

75. Hodgson MC, Astapova I, Hollenberg AN, Balk SP. Activity of androgen receptor antagonist bicalutamide in prostate cancer cells is independent of NCoR and SMRT corepressors. Cancer Res. 2007;67(17):8388-8395.

76. Qin J, et al. Androgen deprivation-induced NCoA2 promotes metastatic and castrationresistant prostate cancer. J Clin Invest. 2014;124(11):5013-5026.

77. Torres-Arzayus MI, et al. High tumor incidence and activation of the PI3K/AKT pathway in transgenic mice define AIB1 as an oncogene. Cancer Cell. 2004;6(3):263-274.

78. Torres-Arzayus MI, Zhao J, Bronson R, Brown M. Estrogen-dependent and estrogen-independent mechanisms contribute to AIB1-mediated tumor formation. Cancer Res. 2010;70(10):4102-4111.

79. Osborne CK, et al. Role of the estrogen receptor coactivator AIB1 (SRC-3) and HER-2/neu in tamoxifen resistance in breast cancer. J Natl Cancer Inst. 2003;95(5):353-361.

80. Smith CL, Nawaz Z, O'Malley BW. Coactivator and corepressor regulation of the agonist/ antagonist activity of the mixed antiestrogen, 4-hydroxytamoxifen. Mol Endocrinol. 1997;11(6):657-666.

81. Munster PN, et al. A phase II study of the histone deacetylase inhibitor vorinostat combined with tamoxifen for the treatment of patients with hormone therapy-resistant breast cancer. $\mathrm{Br} \mathrm{J}$ Cancer. 2011;104(12):1828-1835.

82. Raha P, Thomas S, Thurn KT, Park J, Munster PN. Combined histone deacetylase inhibition and tamoxifen induces apoptosis in tamoxifenresistant breast cancer models, by reversing Bcl-2 overexpression. Breast Cancer Res. 2015;17:26.

83. Wang Y, et al. Bufalin is a potent small-molecule inhibitor of the steroid receptor coactivators SRC-3 and SRC-1. Cancer Res. 2014;74(5):1506-1517.

84. Groner AC, Brown M, Theurillat JP. Targeting transcriptional co-activators in advanced prostate cancer. Cell Cycle. 2016;15(24):3333-3334.

85. Iwafuchi-Doi M, Zaret KS. Pioneer transcription factors in cell reprogramming. Genes Dev. 2014;28(24):2679-2692.
86. Gao N, et al. Forkhead box A1 regulates prostate ductal morphogenesis and promotes epithelial cell maturation. Development. 2005;132(15):3431-3443.

87. Bernardo GM, et al. FOXA1 is an essential determinant of ERalpha expression and mammary ductal morphogenesis. Development. 2010;137(12):2045-2054.

88. Carroll JS, et al. Genome-wide analysis of estrogen receptor binding sites. Nat Genet. 2006;38(11):1289-1297.

89. Wang $Q$, et al. A hierarchical network of transcription factors governs androgen receptor-dependent prostate cancer growth. Mol Cell. 2007;27(3):380-392.

90. Kong SL, Li G, Loh SL, Sung WK, Liu ET. Cellular reprogramming by the conjoint action of $\mathrm{ER} \alpha$, FOXA1, and GATA3 to a ligand-inducible growth state. Mol Syst Biol. 2011;7:526.

91. Carroll JS, et al. Chromosome-wide mapping of estrogen receptor binding reveals long-range regulation requiring the forkhead protein FoxA1. Cell. 2005;122(1):33-43.

92. $\mathrm{He} \mathrm{HH}$, et al. Nucleosome dynamics define transcriptional enhancers. Nat Genet. 2010;42(4):343-347.

93. Lupien M, et al. FoxA1 translates epigenetic signatures into enhancer-driven lineage-specific transcription. Cell. 2008;132(6):958-970.

94. Badve $\mathrm{S}$, et al. FOXA1 expression in breast cancer - correlation with luminal subtype A and survival. Clin Cancer Res. 2007;13(15 pt 1):4415-4421.

95. Jain RK, Mehta RJ, Nakshatri H, Idrees MT, Badve SS. High-level expression of forkhead-box protein A1 in metastatic prostate cancer. Histopathology. 2011;58(5):766-772.

96. Gerhardt J, et al. FOXA1 promotes tumor progression in prostate cancer and represents a novel hallmark of castration-resistant prostate cancer. Am J Pathol. 2012;180(2):848-861.

97. Imamura $Y$, et al. FOXA1 promotes tumor progression in prostate cancer via the insulin-like growth factor binding protein 3 pathway. PLOS One. 2012;7(8):e42456.

98. Mehta RJ, et al. FOXA1 is an independent prognostic marker for ER-positive breast cancer. Breast Cancer Res Treat. 2012;131(3):881-890.

99. Ross-Innes CS, et al. Differential oestrogen receptor binding is associated with clinical outcome in breast cancer. Nature. 2012;481(7381):389-393.

100.Hurtado A, Holmes KA, Ross-Innes CS, Schmidt $D$, Carroll JS. FOXA1 is a key determinant of estrogen receptor function and endocrine response. Nat Genet. 2011;43(1):27-33.

101.Sahu B, et al. Dual role of FoxA1 in androgen receptor binding to chromatin, androgen signalling and prostate cancer. $E M B O J$. 2011;30(19):3962-3976.

102. Wang $\mathrm{D}$, et al. Reprogramming transcription by distinct classes of enhancers functionally defined by eRNA. Nature. 2011;474(7351):390-394.

103. Robinson JL, et al. Elevated levels of FOXA1 facilitate androgen receptor chromatin binding resulting in a CRPC-like phenotype. Oncogene. 2014;33(50):5666-5674.

104.Fu X, et al. FOXA1 overexpression mediates endocrine resistance by altering the ER transcriptome and IL-8 expression in ER-posi- tive breast cancer. Proc Natl Acad Sci U S A. 2016;113(43):E6600-E6609.

105. Quenel-Tueux N, et al. Clinical and genomic analysis of a randomised phase II study evaluating anastrozole and fulvestrant in postmenopausal patients treated for large operable or locally advanced hormone-receptor-positive breast cancer. Br J Cancer. 2015;113(4):585-594.

106.Zhang C, et al. Definition of a FoxA1 Cistrome that is crucial for G1 to S-phase cell-cycle transit in castration-resistant prostate cancer. Cancer Res. 2011;71(21):6738-6748.

107. Hegde NS, Sanders DA, Rodriguez R, Balasubramanian $\mathrm{S}$. The transcription factor FOXM1 is a cellular target of the natural product thiostrepton. Nat Chem. 2011;3(9):725-731.

108. Barbieri CE, et al. Exome sequencing identifies recurrent SPOP, FOXA1 and MED12 mutations in prostate cancer. Nat Genet. 2012;44(6):685-689.

109. Pomerantz MM, et al. The androgen receptor cistrome is extensively reprogrammed in human prostate tumorigenesis. Nat Genet. 2015;47(11):1346-1351.

110.Wang $\mathrm{Q}$, et al. Androgen receptor regulates a distinct transcription program in androgen-independent prostate cancer. Cell. 2009;138(2):245-256.

111. Jin HJ, Zhao JC, Wu L, Kim J, Yu J. Cooperativity and equilibrium with FOXA1 define the androgen receptor transcriptional program. Nat Commun. 2014;5:3972.

112. Ciriello G, et al. Comprehensive molecular portraits of invasive lobular breast cancer. Cell. 2015;163(2):506-519.

113. Wu D, et al. Three-tiered role of the pioneer factor GATA2 in promoting androgen-dependent gene expression in prostate cancer. Nucleic Acids Res. 2014;42(6):3607-3622.

114. Zhao JC, Fong KW, Jin HJ, Yang YA, Kim J, Yu J. FOXA1 acts upstream of GATA2 and AR in hormonal regulation of gene expression. Oncogene. 2016;35(33):4335-4344.

115. He B, et al. GATA2 facilitates steroid receptor coactivator recruitment to the androgen receptor complex. Proc Natl Acad Sci U S A. 2014;111(51):18261-18266.

116. Böhm M, Locke WJ, Sutherland RL, Kench JG, Henshall SM. A role for GATA-2 in transition to an aggressive phenotype in prostate cancer through modulation of key androgen-regulated genes. Oncogene. 2009;28(43):3847-3856.

117. Taylor BS, et al. Integrative genomic profiling of human prostate cancer. Cancer Cell. 2010;18(1):11-22.

118. Vidal SJ, et al. A targetable GATA2-IGF2 axis confers aggressiveness in lethal prostate cancer. Cancer Cell. 2015;27(2):223-239.

119. Wu JD, Haugk K, Woodke L, Nelson P, Coleman I, Plymate SR. Interaction of IGF signaling and the androgen receptor in prostate cancer progression. J Cell Biochem. 2006;99(2):392-401.

120. Eeckhoute J, Keeton EK, Lupien M, Krum SA, Carroll JS, Brown M. Positive cross-regulatory loop ties GATA-3 to estrogen receptor alpha expression in breast cancer. Cancer Res. 2007;67(13):6477-6483.

121. Theodorou V, Stark R, Menon S, Carroll JS. GATA3 acts upstream of FOXA1 in mediating ESR1 binding by shaping enhancer accessibility. 
Genome Res. 2013;23(1):12-22.

122.Parikh P, Palazzo JP, Rose LJ, Daskalakis C, Weigel RJ. GATA-3 expression as a predictor of hormone response in breast cancer. J Am Coll Surg. 2005;200(5):705-710.

123. Stephens PJ, et al. The landscape of cancer genes and mutational processes in breast cancer. Nature. 2012;486(7403):400-404.

124. Banerji S, et al. Sequence analysis of mutations and translocations across breast cancer subtypes. Nature. 2012;486(7403):405-409.

125. Ellis MJ, et al. Whole-genome analysis informs breast cancer response to aromatase inhibition. Nature. 2012;486(7403):353-360.

126.Usary J, et al. Mutation of GATA3 in human breast tumors. Oncogene. 2004;23(46):7669-7678.

127. Mair B, et al. Gain- and loss-of-function mutations in the breast cancer gene GATA3 result in differential drug sensitivity. PLoS Genet. 2016;12(9):e1006279.

128. Adomas AB, Grimm SA, Malone C, Takaku M, Sims JK, Wade PA. Breast tumor specific mutation in GATA3 affects physiological mechanisms regulating transcription factor turnover. $B M C$ Cancer. 2014;14:278.

129. Bates DL, Chen Y, Kim G, Guo L, Chen L. Crystal structures of multiple GATA zinc fingers bound to DNA reveal new insights into DNA recognition and self-association by GATA. J Mol Biol. 2008;381(5):1292-1306.

130. Jiang YZ, Yu KD, Zuo WJ, Peng WT, Shao ZM. GATA3 mutations define a unique subtype of luminal-like breast cancer with improved survival. Cancer. 2014;120(9):1329-1337.

131. Pereira B, et al. The somatic mutation profiles of 2,433 breast cancers refines their genomic and transcriptomic landscapes. Nat Commun. 2016;7:11479. 132.Zhang X, Cowper-Sal lari R, Bailey SD, Moore
$\mathrm{JH}$, Lupien M. Integrative functional genomics identifies an enhancer looping to the SOX9 gene disrupted by the $17 \mathrm{q} 24.3$ prostate cancer risk locus. Genome Res. 2012;22(8):1437-1446.

133. Cowper-Sal lari R, et al. Breast cancer risk-associated SNPs modulate the affinity of chromatin for FOXA1 and alter gene expression. Nat Genet. 2012;44(11):1191-1198.

134. Varambally S, et al. The polycomb group protein $\mathrm{EZH} 2$ is involved in progression of prostate cancer. Nature. 2002;419(6907):624-629.

135. Kleer CG, et al. EZH2 is a marker of aggressive breast cancer and promotes neoplastic transformation of breast epithelial cells. Proc Natl Acad SciU S A. 2003;100(20):11606-11611.

136. Xu K, et al. EZH2 oncogenic activity in castration-resistant prostate cancer cells is Polycomb-independent. Science. 2012;338(6113):1465-1469. 